\title{
Role of Non-coding RNAs in Fungal Pathogenesis and Antifungal Drug Responses
}

\author{
Sourabh Dhingra ${ }^{1}$
}

Accepted: 4 August 2020 / Published online: 2 October 2020

(C) The Author(s) 2020

\begin{abstract}
Purpose of Review Non-coding RNAs (ncRNAs), including regulatory small RNAs (sRNAs) and long non-coding RNAs (IncRNAs), constitute a significant part of eukaryotic genomes; however, their roles in fungi are just starting to emerge. ncRNAs have been shown to regulate gene expression in response to varying environmental conditions (like stress) and response to chemicals, including antifungal drugs. In this review, I highlighted recent studies focusing on the functional roles of ncRNAs in pathogenic fungi.

Recent Findings Emerging evidence suggests sRNAs (small RNAs) and lncRNAs (long non-coding RNAs) play an important role in fungal pathogenesis and antifungal drug response. Their roles include posttranscriptional gene silencing, histone modification, and chromatin remodeling. Fungal pathogens utilize RNA interference (RNAi) mechanisms to regulate pathogenesisrelated genes and can also transfer sRNAs inside the host to suppress host immunity genes to increase virulence. Hosts can also transfer sRNAs to induce RNAi in fungal pathogens to reduce virulence. Additionally, sRNAs and lncRNAs also regulate gene expression in response to antifungal drugs increasing resistance (and possibly tolerance) to drugs.

Summary Herein, I discuss what is known about ncRNAs in fungal pathogenesis and antifungal drug responses. Advancements in genomic technologies will help identify the ncRNA repertoire in fungal pathogens, and functional studies will elucidate their mechanisms. This will advance our understanding of host-fungal interactions and potentially help develop better treatment strategies.
\end{abstract}

Keywords siRNA $\cdot$ miRNAs $\cdot$ RNAi $\cdot \operatorname{lncRNAs} \cdot$ Fungal pathogenesis $\cdot$ Drug response

\section{Introduction}

Fungi are a major contributor to animal and plant pathogenesis and crop loss [1]. It is estimated that fungi cause 1.6 million deaths, and over one billion people are affected by fungal infections [2]. Global warming and climate change have resulted in increased incidences of fungal infections, with some animal species on the verge of extinction [3]. Reverse and forward genetic approaches have focused on protein-coding genes to identify molecular mechanisms associated with fungal pathogenesis; however, mortality rates are still high, and

This article is part of the Topical Collection on Mycology

Sourabh Dhingra

sdhingr@clemson.edu

1 Department of Biological Sciences; Eukaryotic Pathogen Innovation Center, Clemson University, 190 Collings St, Life Sciences Building, Clemson, SC, USA crop losses are increasing [1, 2]. The central dogma, DNA $\rightarrow$ mRNA $\rightarrow$ protein, explains protein as a functional unit [4]; however, it exhibits incomplete information regarding gene regulation, timing, and rates of protein production and fails to explain organismal complexity, referred to as the G-value paradox [5]. Recent genomic advances have identified that only $2-3 \%$ of the transcriptome is translated into proteins $[6$, 7], thus indicating potential widespread roles of untranslated transcripts or non-coding RNAs (ncRNAs) [8]. Interestingly, organismal complexity highly correlates with the number of ncRNAs but not protein-coding genes [9].

ncRNAs have been identified in most eukaryotic genomes $[10,11]$, prokaryotic genomes $[12,13]$, and in archaea [14, 15]. They can be classified based on their functional roles as either structural ncRNAs including transfer RNAs, ribosomal RNAs, small nuclear and nucleolar RNAs, or regulatory RNAs including small RNAs (sRNAs) and long ncRNAs (lncRNAs) (> 200 nucleotides) [16] including natural antisense transcripts (NATs) [17]. In eukaryotes, sRNAs can be 
further classified as short-interfering RNAs (siRNAs), microRNAs (miRNAs), and Piwi-interacting RNAs [18].

siRNAs and miRNAs are both small ncRNAs $(20-30 \mathrm{nt}$ long); however, siRNAs stem from exogenous dsRNAs (e.g., viruses, transposons, or transgene) and are excised from completely complementary target sequences, whereas miRNAs are endogenously expressed, processed from precursors that form stem loops, and are not fully complementary to the target. Once processed, both siRNA and miRNAs form active complexes called RNA-induced silencing complexes (RISC) to silence genes in a process called RNA interference (RNAi) [19].

The components of RNAi machinery consist of RNAdependent RNA polymerase (RdRP) [20], ribonuclease enzyme, Dicer [21], and the small RNA-binding partner, Argonaute proteins [22]. In the model fungus, Neurospora crassa, where RNAi was first discovered in fungi [23], RdRP has both RNA-dependent and DNA-dependent (DdRP) RNA polymerase activity with DdRP activity much stronger than RdRP [24, 25]. Once aberrant DNA (aDNA) is detected in N. crassa, a helicase unwinds the DNA and RdRP with its DdRP activity synthesizes ssRNA and then using RdRP activity to form dsRNA [24]. Thus, using DdRP activity, the fungus can differentiate endogenous RNAs and is specific to targets originating from aDNA production [20]. $N$. crassa has two Dicer-like enzymes with overlapping roles, which bind to dsRNA and cleave them into 20-25 nt long sRNAs [26]. These sRNAs bind to Argonaute proteins and form inactive RISC complex [27]. The Argonaute protein nicks the non-targeting strand and recruits additional exonuclease, QIP, to degrade it, leading to activation of the RISC complex [28]. Based on sequence complementarity of sRNA, the RISC complex is targeted, leading to posttranscriptional gene silencing. These core RNAi components (RdRP, Dicer, and Argonaute proteins) have been identified in the majority of eukaryotes, including pathogenic fungi. Notably, Saccharomyces cerevisiae lacks Dicer and Argonaute proteins; however, expression of genes encoding these proteins from $S$. castelli can activate RISC-mediated gene silencing in S. cerevisiae [29]. This cross-species complementation study indicates the presence of some RNAi machinery components that are functional in $S$. cerevisiae. Interestingly, in pathogenic fungi Cryptococcus deuterogattii and Ustilago maydis, loss of RNAi has been shown to correlate with the loss of active fulllength retroelements and shorter centromeres [30]. Given that RNAi is present in most eukaryotes, it is possible that this defense mechanism was present in a common ancestor and, in the absence of selection pressure, including loss of fulllength transposable elements, subsequently became dispensable in some fungi [29-32].

Piwi-interacting RNAs are mostly identified in metazoan genomes due to their association with Argonaute proteins belonging to the Piwi clade [33] and are therefore not discussed in this review. On the other hand, lncRNAs are > $200 \mathrm{nt}$ long and have been shown to play a critical role in diseases like cancer, Alzheimer's disease, diabetes, and cardiovascular and neurological disorders [34, 35]; however, functional studies on the role of lncRNAs are lacking in fungi [36]. Additionally, unlike protein-coding genes, there is a lack of sequence conservation across species that makes identification and functional prediction hard (if not impossible) for these ncRNAs [37]. It is important to note that the structure of lncRNAs determines its function and lack of sequence conservation does not mean lack of function [37]. Interestingly, the promoter regions of these lncRNAs show high degrees of sequence similarity across species [38]. However, both the identification and regulatory roles of lncRNAs in pathogenic fungi are lacking. IncRNAs are generally transcribed from RNA pol II, can be polyadenylated or not, and may or may not include a $5^{\prime}$ cap and function in cis or trans. IncRNAs have a wide variety of functions, including transcriptional interference, chromatin remodeling, and histone modifications [39-42].

Another form of regulatory lncRNAs - natural antisense transcripts (NATs) - is commonly found in eukaryotic genomes and can range from hundreds to thousands of nucleotides. They can be transcribed in sense or antisense direction and form complementary base pairs with $5^{\prime}$ or $3^{\prime}$ end of the coding transcript [43, 44]. NATs have widespread mechanisms of controlling gene expression by forming a senseantisense pair and blocking other factors or by remodeling chromatin $[44,45]$. This review will focus on recent advances on the roles of ncRNAs, including sRNAs and their role in RNAi, lncRNAs, and NATs in fungal pathogenesis and antifungal drug responses.

\section{Role of ncRNAs in Fungal Pathogenesis}

\section{Role of sRNAs and RNAi in Fungal Pathogenesis}

Fungi imperfecti Verticillium dahliae, the causative agent of verticillium wilt, expresses core RNAi machinery [46]. sRNAs detected in $V$. dahliae are processed from 70 to $400 \mathrm{bp}$ long precursors into 18-20 nt in length with G preferred at position 1; however, the exact mechanism of this processing is unknown [47•]. Blotting analysis to identify highly expressed sRNAs identified only one RNA pol II expressed sRNA-Vd mi-like RNA 1, VdmIIR1 (processed from precursor VdMILR1), which regulates fungal pathogenesis by chromatin remodeling. Interestingly, the deletion of canonical Dicer proteins, which are involved in cleaving precursors, increased the expression of VdmIIR1, indicating that canonical dicers do not play a role in the $V$. dahliae RNAi mechanism. Further analysis revealed the presence of a third RNAse III domain-containing protein, named Vdr3, whose 
expression directly correlated with VdmilRNA1 expression [47•]. Canonical Dicer proteins contain two RNAse III domains and a PAZ domain (Piwi/Argonaute/Zwille domain); however, VdR3 consists only of a single RNAse III domain $[21,47 \bullet]$. Interestingly, budding yeasts $S$. castellii and Kluyveromyces also contain a non-canonical single RNAse III containing a functional dicer protein, $d c r-1[29,48]$. It is fair to assume that fungal systems have evolved to express non-canonical RNAi components, possibly due to selective pressures that may include host-pathogen interactions and warrants future investigation.

Comparative mRNA analysis identified an uncharacterized protein-coding gene, VdHy1, as a target for milR1 with predicted sRNA-binding site in 3' UTR. VdmilR1 repressed the transcription but did not cleave VdhY1, which is a positive regulator of virulence. Chromatin regulation by histone modification is known to cause changes in transcription levels [49, 50], and methylation of histones ( $\mathrm{H} 3 \mathrm{~K} 9 \mathrm{me} 3$ ) is a classical hallmark of heterochromatin formation [51, 52]. H3K9me3 antibody-mediated chromatin immunoprecipitation (ChIP) followed by quantitative PCR identified heterochromatin enrichment in overexpression (OE) VdMILR1 (precursor) and less enrichment in $\Delta v d m i l R 1$ strains [47॰]. Surprisingly, $\mathrm{OE}$ of VdR3, which increased milR1 levels, showed increased virulence. As VdR3 is indispensable for V. dahliae and functions as a non-canonical RNAse III domain protein, it is likely that VdR3 has additional, yet unknown functions in fungal virulence [47•]. More studies are needed to understand the role of non-canonical Dicer-like proteins in regulating gene expression and RNA processing in fungal pathogenesis.

Additionally, host miRNA-mediated gene silencing has also been shown to regulate $V$. dahliae virulence. Sequencing analysis in fungi recovered from cotton in planta infection but not in vitro cultures revealed higher accumulation of host miRNAs, miR166 and miR159, indicating host control of fungal RNAi machinery [53••]. Target analysis revealed binding sites of miR166 and miR159 in the $\mathrm{Ca}^{2+}$-dependent calcium protease calpain, clp-1, and isotrichodermin C-15 hydroxylase, HiC-15, respectively; null mutants of both genes show significant virulence defects. Moreover, sequences of Clp-1 and HiC-15 are conserved, especially in miRNA-binding regions in various strains of $V$. dahliae, and mir166 Clp-1 silencing also plays a role in tomato infections by VdLs.17 strain [53・•]. Even though it was evident that miRNAs can lead to cross-species gene silencing, it was unclear how sRNAs were transferred from host to pathogen. Recently, Jin et al. identified Arabidopsis thaliana sRNAs in a careful examination of isolated Botrytis cinerea cells from infected plants, many of which can target the $B$. cinerea genome. $A$. thaliana secretes exosome-like selective sRNA containing extracellular vesicles (EVs), which are taken up by fungi [54••]. In support of EVs-mediated cross-kingdom transfer of sRNAs, plant EVs-specific marker tetraspanins TET8 and
TET9 are accumulated at the site of infection, and tet 8 and tet 9 mutants showed increase susceptibility to $B$. cinerea infection [54・•, 55].

Interestingly, the opposite phenomenon of sRNA transfer from pathogen to host has also been shown to regulate virulence. Recently, a similar EVs-dependent cross-kingdom transfer of sRNA was observed in a mosquito fungal pathogen Beauveria bassiana. Bba-milR1, a dicer-dependent sRNA, plays a dual role in pathogenesis by silencing host immune genes to initiate infection and preventing immune recognition for fungal proliferation. To initiate infection, B. bassiana upregulates bba-milR1 expression, which gets transported to the host via EVs and silences toll immune pathways by binding to toll ligand Spätzle, Spz4. This effect is mediated by host Argonaute protein, Ago1. This initial response helps the fungus invade the cell hemolymph [56॰]. Melanization is a key immune pathway in invertebrates resulting from the detection of microbes in the hemocoel. Once a pathogen is detected, melanin can encapsulate pathogens by forming crosslinks with microbial surface pathogen-associated molecular patterns (PAMPs) [57]. In mosquitos, melanization is regulated by the serine protease CLIPB9 that cleaves prophenoloxidase (PPO) into active phenoloxidase (PO), a key enzyme in the melanization process [58]. B. bassiana bba-milR1 interacts with and induces CLIPB9 expression. However, once in hemolymph, B. bassiana downregulates expression of bbamilR1 preventing CLIPB9-mediated cleavage of PPO to PO to inhibit melanization allowing fungal proliferation [56 $6^{\bullet}$. It is interesting to note that host hijacking of the fungal virulence machinery is preserved during evolution. One possible reason can be a long-term survival strategy by fungi to keep the host alive by downregulating its virulence during infection.

Similarly, B. cinerea in planta infection of A. thaliana and Solanum lycospericum (tomato) revealed enrichment of $73 \mathrm{Bc}$ sRNAs compared with in vitro cultures, the majority of which can target the plant genome [59・•]. Specifically, mitogenactivated protein kinase 2 (MPK2) and MPK1, oxidative stress-related gene peroxiredoxin (PRXIIF), and cell wallassociated kinase (WAK) - all of which play a role in plant immunity against fungal infection-were targeted by $\mathrm{Bc}$ sRNAs, siR3.2, siR3.1, and siR-5, respectively, and are mediated by host Argonaute protein, Ago1. Additionally, Bc sRNAs expressing transgenic $A$. thaliana lines were hypervirulent when infected with $B$. cinerea, a phenotype that can be reversed by destroying sRNA target sites [59••] confirming that fungal sRNAs regulate virulence by suppressing host immunity genes.

Levels of ncRNAs are differentially regulated in both host and fungi upon infection of the host in many pathogenic fungi [60]. Further research is needed to identify the regulatory roles of these ncRNAs in response to fungal infections. What is unclear is if these pathways can regulate cross-kingdom RNAi or rather the presence of additional components to mediate host-pathogen interactions. 
It is important to note that functional RNAi systems have been identified in major human fungal pathogens Candida albicans [61], Cryptococcus neoformans [62], and Aspergillus fumigatus [63] and major crop pathogen Magnaporthe oryzae [64] and Fusarium spp. [65]; however, their role in virulence of these fungal pathogens remains to be fully explored. Interestingly, in fungi lacking core RNAi components, active transposition of shorter retroelements may provide a pathogenic advantage; however, a direct correlation between loss of RNAi and virulence is not known at this time [30, 31]. Further research will elucidate the full potential of RNAi-associated mechanisms or evolutionary lack thereof in important fungal pathogens.

\section{Roles of Long ncRNAs (IncRNAs) in Fungal Pathogenesis}

Functional analyses of lncRNAs in pathogenic fungi are limited. In the human pathogenic fungus, C. neoformans, a random mutagenesis screen identified the long ncRNA RZE1 as a regulator of Znf2-a yeast to hyphae morpho switch regulator $[66,67]$. Deletion of $z n f 2$ locks cells in the pathogenic yeast form and increases its virulence [67]. Mechanistically, RZE1 is a nuclear localized lncRNA that reduces the cytoplasmic accumulation of znf2 mRNA [66]. It is unclear if decreased cytoplasmic localization or increased nuclear localization is due to increased cytoplasmic degradation or reduced transport; however, this mislocalization possibly affects Znf2 protein levels. Although direct role(s) of RZE1 in fungal pathogenesis are not described to date, it can be speculated that $\mathrm{OE}$ RZE1 will lead to hypervirulence (correlate with $\Delta$ Znf2 strain) and $\triangle$ RZE1 to be hypovirulent (correlate with $\mathrm{OE}$ Znf2 strain) [67]. Further research is needed to test these hypotheses.

In the plant pathogen, Ustilago maydis, a developmentally regulated lncRNA, ncRNA1, has been identified with a regulatory role in the expression of natural antisense transcripts (see the next section for details) and pathogenesis [68•, 69]. Interestingly, ectopically complementing ncRNA1 under the influence of a constitutive promoter in $\Delta$ ncRNA1 strain did not complement the pathogenesis phenotype suggesting in cis roles in pathogenesis [68•, 69]. The location-specific function might be due to regulatory elements present at that genomic locus, or the constitutive promoter uncouples development and stress-specific regulation leading to loss of function. Further research is needed to identify the regulation of ncRNA1 in plant fungal pathogenesis. A gene, in trans, UMAG 12316, shares sequence similarity with ncRNA1, and a $\triangle U M A G 12316$ strain showed reduced virulence, however, not as severe reduction as $\Delta$ ncRNA1 $[68 \bullet, 70]$. Given that ncRNA1 is a negative regulator of $U M A G \_12316$ transcript levels, it is possible that stochiometric levels of $U M A G \quad 12316$ are important for pathogenesis or ncRNA1 has other yet unidentified targets with a potential role in pathogenesis.

Non-sense mediated decay (NMD) pathway plays an important role in clearing aberrant mRNAs to protect cells from errors of RNA biogenesis and preventing protein mutations [71]. However, it is unclear if the NMD pathway recognizes ncRNAs and plays an important role in maintaining their levels [71, 72]; however, its role in fungal pathogenesis is limited. In $S$. cerevisiae, the NMD pathway is controlled by Xrn1 exonuclease that destabilizes Xrn1-sensitive lncRNAs [73]. In entomopathogenic fungi, Cordyceps militaris, deletion of the NMD pathway regulator $x r n 1$ results in a significant virulence defect [74]. Further analysis revealed upregulation of 10 lncRNAs and downregulation of 8 of their target genes controlling virulence and sexual development. Even though not direct, this study shows the potential role of IncRNAs in pathogenesis and their regulation by the NMD pathway in $C$. militaris [72].

As we are in the genomic revolution and the quality of fungal genomes is improving, future research will identify additional lncRNAs in fungi, and functional analyses are expected to reveal diverse roles for lncRNAs in gene regulation, chromatin remodeling, and fungal pathogenesis. Given that chromatin remodeling and gene regulation play a major role in fungal development process and secondary metabolite production, it is easy to speculate on their potential roles in pathogenesis and host-pathogen interactions.

\section{Role of Natural Antisense Transcripts in Fungal Pathogenesis}

NATs are widely present in eukaryotic genomes and can be expressed in sense or antisense direction to the ORF. They usually form a sense-antisense pair by complementary base pairing; however, their roles in gene regulation are more complex [45]. In the fungal plant pathogen U. maydis, ncRNAs and NATs are expressed in similar numbers as mRNAs, indicating very widespread roles of ncRNAs in this pathogen [75]. Interestingly, like $S$. cerevisiae canonical components of RNAi, viz., RdRP, Dicer, and Argonaute proteins are absent in this fungus [76]. Functional analysis of one genomic locus identified two NATs asUMAG_02150 and as-UMAG_02151 (expressed at the same locus as ncRNA1 (discussed above)) with functional roles in gene regulation, teliospore formation, and pathogenesis [68•, 69]. As NATs as-UMAG 02150 and as-UMAG 02151 are both synthesized in the antisense direction overlapping ORF UMAG_02150 and UMAG_02151, respectively, it is not possible to study the effects of one deletion without impacting other. Regulatory study identified a region upstream of as-UMAG_02151 termed as $\mathrm{P}_{\text {as-UMAG } 02151 \text {, as a }}$ 
negative regulator of as-UMAG_02150 and asUMAG_02151 expression and a positive regulator of virulence $[68 \cdot, 69]$. Ectopic complementation of $\mathrm{P}_{\text {as-UMAG_02151 }}$ did not complement the pathogenesis defect of the deletion mutant strongly indicating the role(s) of regulatory control at this genomic locus. Interestingly, deletion of the proteincoding gene UMAG_02151 ( $3^{\prime}$ end of gene overlaps as$U M A G-02151)$ did not affect virulence, whereas $U M A G \_02150$ deletion (which overlaps as-UMAG_0215) reduced virulence; however, functional characterization failed to establish a direct role of as-UMAG_02150 in pathogenesis. UMAG_02150 is a xylitol hydrogenase and is necessary to use xylitol as the sole carbon source. Ectopic expression of UMAG_02150 complemented the xylose growth phenotype of the deletion mutant but did not complement virulence defect, indicating a locus-specific

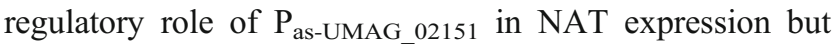
not ORF function [68•]. Importantly, UMAG_12316 (a gene regulated by ncRNA1 as mentioned in the previous section) expression was altered in $\triangle U M A G \_02150$ strain. Further research is needed to evaluate direct or indirect roles of as-UMAG_02150 in regulating UMAG_12316 expression and if these are mediated by IncRNA, ncRNA1.

In another plant, pathogen A. flavus, genome-wide analysis identified temperature-regulated expression of NATs, with a NAT antisense to aflD gene upregulated at $37^{\circ} \mathrm{C}$ [77]. aflD gene encodes a protein in the biosynthetic gene cluster of virulence factor, aflatoxin, that is highly produced at $28^{\circ} \mathrm{C}$ but is repressed at $37^{\circ} \mathrm{C}$ [78]. It is possible but unclear if aflD NAT regulates temperature-dependent expression of aflatoxin $[77,79]$. Further research is needed to understand the complex roles of NATs in fungal pathogenesis and toxin production.

\section{Role of ncRNAs in the Antifungal Drug Response}

\section{Role of RNAi in Fungal Drug Response}

ncRNAs have been implicated in drug response in many different forms of cancers and function by regulating proteincoding genes, signaling pathways, cell death pathways, and drug efflux [80], with less known about their roles in fungi. In the human fungal pathogen, Mucor circinelloides, a spontaneous unstable and reversible RNAi-based drug resistance mechanism has been identified and termed as epimutation $[81 \bullet \bullet, 82 \bullet]$. M. circinelloides expresses both canonical and non-canonical RNAi machinery to induce endogenous RNA and exogenous transgene-dependent gene silencing [83]. The antifungal drug FK506 interacts with and forms a complex with the FKBP12 gene product, $f k b A$ (peptidylprolyl isomerase), inhibiting the highly conserved protein phosphatase calcineurin [84]. Mutations in either $f k b A$ or calcineurin genes confer resistance to FK506. However, transient mutant strains or epimutants, with no mutations in $f k b A$ or calcineurin genes, were identified. In these epimutants, endogenous sRNA derived from an mRNA precursor silences the $f k b A$ gene [81••]. Interestingly, strains lacking the dicer enzyme $d c l 2$, argonaute protein ago 1, or RNA-dependent RNA polymerase $r d r p 2-$ all components of RNAi core machinery-did not produce any epimutants $[81 \bullet \bullet, 85]$, indicating an important role of RNAi in acquiring transient fungal drug resistance and epimutations. A similar mechanism for 5-fluoroorotic acid (5-FOA) resistance, a different class of molecule, has been reported in M. circinelloides suggesting a conserved RNAi mechanism to regulate xenobiotic stress-mediated gene expression [82•]. This phenotypic plasticity allows an organism to rapidly adapt to a given stress; however, given its reversible nature allows the organism to revert to "wild-type" phenotype in a stress-free environment without introducing genetic mutations in the presence of the stress, which may or may not be beneficial long term. It will be interesting to see if in the presence of drugs, endogenous sRNAs are generated from mRNAs of drug target genes in other fungal pathogens and if this mechanism is conserved to mitigate additional stresses observed during host-pathogen interactions and infections.

\section{Role of IncRNAs in Fungal Drug Response}

A major study in the fission yeast $S$. pombe identified a role for a lncRNA, ncRNAtgp1, in drug sensitivity against a wide variety of drugs [86•*]. Deletion of ncRNAtgp1 resulted in > 50-fold increase in mRNA levels of the neighboring gene, tgp1, while other neighboring genes expression was unaltered, suggesting a role in cis. Functionally, ncRNAtgp 1 alters the nucleosome density upstream of tgp 1 , hindering binding of transcription factor $\mathrm{Pho} 7$ in the presence of phosphate, thus

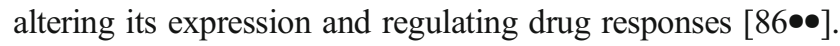
This shows the complex role of lncRNAs in gene regulation to mediate stress-specific functions. Given conserved synteny of this lncRNA in other Schizosaccharomyces species [86••, 87], further research will elucidate if IncRNA-mediated gene regulation is a conserved mechanism.

Chromatin modification involving histone acetylation and deacetylation has also been shown to play a role in the fungal drug response in many fungal species [85]; however, it is unclear at this time if this is mediated by ncRNAs. This represents an interesting area of future research. Interestingly, upon fungal infection, differential ncRNAs are detected in host cells [60]. Recent reports indicate roles of ncRNAs in host cell regulation of drug-metabolizing enzymes CYP450, UDP-glucuronosyltransferase, and drug transporters [88]. CYP450 enzymes represent an important class of enzymes responsible for metabolizing the azole class of antifungal drugs [89]. As evident from the examples above that the host and pathogen can regulate a cross-kingdom sRNA response, 
future research will seek to uncover if fungal interactions with the host cells cause differential ncRNA-mediated drug metabolism and responses to alter disease outcomes.

\section{Conclusions and Perspectives}

In this review, the known roles of ncRNAs in fungi and fungal pathogenesis and drug response are described. ncRNAs have widespread functions, including indirect transcriptional regulation by recruiting regulatory protein complex or methylation of specific genes and by directly regulating histone modifications or other chromatin regulatory complexes to modify chromatin structure [20, 90, 91]. Even though a lot is known about ncRNAs in mammalian cells, more work is needed to understand their functional roles in pathogenic fungi. We have gained many insights from deep sequencing analyses of fungal genomes and transcriptomes; however, identification of ncRNAs in fungal genomes is still challenging. For example, in A. fumigatus, ncRNAs have only been detected to date by a complementary cDNA library approach and not by advanced sequencing technologies [92]. Furthermore, a majority of fungal transcriptome studies has utilized polyA+ based mRNA sequencing; however, it is clear that fungal lncRNAs are not necessarily polyadenylated; thus, they are not detected/identified. As the sequence similarity for ncRNAs is low, advancements in genomics technologies will help uncover the ncRNA repertoire in fungal pathogens. This will still require functional studies to understand the complex roles of ncRNAs (lncRNA and sRNAs) in fungal pathogenicity and drug response. Recently, drug tolerance, which is distinct from drug resistance, has been described in C. albicans [93]. Given that ncRNAs play an important role in stress responses, including antifungal drugs $[81 \bullet \bullet, 82 \bullet]$, it is tempting to speculate that tolerance phenotypes are mediated in part by ncRNAs; however, functional studies are needed to test this hypothesis. As drug tolerance can lead to drug resistance [94], understanding the roles of ncRNAs in fungal drug response is crucial in designing new treatment strategies. These studies in pathogenic fungi will not only tell us the specific roles of ncRNAs in pathogenesis and drug response but will also add to our understanding of molecular mechanisms involved in host-pathogen interactions.

\section{Compliance with Ethical Standards}

Conflict of Interest The authors declare that they have no conflict of interest.

Human and Animal Rights and Informed Consent This article does not contain any studies with human or animal subjects performed by any of the authors.

Open Access This article is licensed under a Creative Commons Attribution 4.0 International License, which permits use, sharing, adaptation, distribution and reproduction in any medium or format, as long as you give appropriate credit to the original author(s) and the source, provide a link to the Creative Commons licence, and indicate if changes were made. The images or other third party material in this article are included in the article's Creative Commons licence, unless indicated otherwise in a credit line to the material. If material is not included in the article's Creative Commons licence and your intended use is not permitted by statutory regulation or exceeds the permitted use, you will need to obtain permission directly from the copyright holder. To view a copy of this licence, visit http://creativecommons.org/licenses/by/4.0/.

\section{References}

Papers of particular interest, published recently, have been highlighted as:

- Of importance

- Of major importance

1. Almeida F, Rodrigues ML, Coelho C. The Still Underestimated Problem of Fungal Diseases Worldwide. Front Microbiol. 2019;10:214. https://doi.org/10.3389/fmicb.2019.00214.

2. Brown GD, Denning DW, Gow NA, Levitz SM, Netea MG, White TC. Hidden killers: human fungal infections. Sci Transl Med. 2012;4(165):165rv13. https://doi.org/10.1126/scitranslmed. 3004404.

3. Casadevall A. Don't forget the fungi when considering global catastrophic biorisks. Health Security. 2017;15(4):341-2. https://doi. org $/ 10.1089 / \mathrm{hs} .2017 .0048$.

4. Crick F. Central dogma of molecular biology. Nature. 1970;227(5258):561. https://doi.org/10.1038/227561a0.

5. Hahn MW, Wray GA. The g-value paradox. Evol Dev. 2002;4(2): 73-5. https://doi.org/10.1046/j.1525-142X.2002.01069.x.

6. Mattick JS. Non-coding RNAs: the architects of eukaryotic complexity. EMBO Rep. 2001;2(11):986-91. https://doi.org/10.1093/ embo-reports/kve230.

7. Kaikkonen MU, Lam MTY, Glass CK. Non-coding RNAs as regulators of gene expression and epigenetics. Cardiovasc Res. 2011;90(3):430-40. https://doi.org/10.1093/cvr/cvr097.

8. Herbert A. The four Rs of RNA-directed evolution. Nat Genet. 2004;36(1):19-25. https://doi.org/10.1038/ng1275.

9. Perkins DO, Jeffries C, Sullivan P. Expanding the 'central dogma': the regulatory role of nonprotein coding genes and implications for the genetic liability to schizophrenia. Mol Psychiatry. 2005;10(1): 69-78. https://doi.org/10.1038/sj.mp.4001577.

10. Dinger ME, Amaral PP, Mercer TR, Mattick JS. Pervasive transcription of the eukaryotic genome: functional indices and conceptual implications. Brief Funct Genomics. 2009;8(6):407-23. https:// doi.org/10.1093/bfgp/elp038.

11. Prasanth KV, Spector DL. Eukaryotic regulatory RNAs: an answer to the 'genome complexity' conundrum. Genes Dev. 2007;21(1): 11-42. https://doi.org/10.1101/gad.1484207.

12. Stav S, Atilho RM, Arachchilage GM, Nguyen G, Higgs G, Breaker RR. Genome-wide discovery of structured noncoding RNAs in bacteria. BMC Microbiol. 2019;19:66. https://doi.org/ 10.1186/s12866-019-1433-7.

13. Repoila F, Darfeuille F. Small regulatory non-coding RNAs in bacteria: physiology and mechanistic aspects. Biol Cell. 2009;101(2): 117-31. https://doi.org/10.1042/bc20070137.

14. Buddeweg A, Daume M, Randau L, Schmitz RA. Noncoding RNAs in archaea: genome-wide identification and functional classification. High-density sequencing applications in microbial 
molecular genetics. Methods Enzymol. 2018;612:413-42. https:// doi.org/10.1016/bs.mie.2018.08.003.

15. Gelsinger DR, DiRuggiero J. The Non-Coding Regulatory RNA Revolution in Archaea. Genes (Basel). 2018;9(3):141. https://doi. org/10.3390/genes 9030141 .

16. Gil N, Ulitsky I. Regulation of gene expression by cis-acting long non-coding RNAs. Nat Rev Genet. 2020;21(2):102-17. https://doi. org/10.1038/s41576-019-0184-5.

17. Faghihi MA, Wahlestedt C. Regulatory roles of natural antisense transcripts. Nat Rev Mol Cell Biol. 2009;10(9):637-43. https://doi. org $/ 10.1038 / \mathrm{nrm} 2738$.

18. Chu CY, Rana TM. Small RNAs: regulators and guardians of the genome. J Cell Physiol. 2007;213(2):412-9. https://doi.org/10. 1002/jcp. 21230 .

19. Carthew RW, Sontheimer EJ. Origins and mechanisms of miRNAs and siRNAs. Cell. 2009;136(4):642-55. https://doi.org/10.1016/j. cell.2009.01.035.

20. Dang YK, Yang QY, Xue ZH, Liu Y. RNA interference in fungi: pathways, functions, and applications. Eukaryot Cell. 2011;10(9): 1148-55. https://doi.org/10.1128/ec.05109-11.

21. Song MS, Rossi JJ. Molecular mechanisms of dicer: endonuclease and enzymatic activity. Biochem J. 2017;474(10):1603-18. https:// doi.org/10.1042/bcj20160759.

22. Meister G. Argonaute proteins: functional insights and emerging roles. Nat Rev Genet. 2013;14(7):447-59. https://doi.org/10.1038/ $\operatorname{nrg} 3462$.

23. Romano N, Macino G. Quelling - transient inactivation of geneexpression in Neurospora crassa by transformation with homologous sequences. Mol Microbiol. 1992;6(22):3343-53. https://doi. org/10.1111/j.1365-2958.1992.tb02202.x.

24. Lee H-C, Aalto AP, Yang Q, Chang S-S, Huang G, Fisher D, et al. The DNA/RNA-Dependent RNA Polymerase QDE-1 Generates Aberrant RNA and dsRNA for RNAi in a Process Requiring Replication Protein A and a DNA Helicase. PLoS Biol. 2010;8(10):e1000496. https://doi.org/10.1371/journal.pbio. 1000496.

25. Makeyev EV, Bamford DH. Cellular RNA-dependent RNA polymerase involved posttranscriptional gene silencing has two distinct activity modes. Mol Cell. 2002;10(6):1417-27. https://doi.org/10. 1016/s1097-2765(02)00780-3.

26. Catalanotto C, Pallotta M, ReFalo P, Sachs MS, Vayssie L, Macino $\mathrm{G}$, et al. Redundancy of the two dicer genes in transgene-induced posttranscriptional gene silencing in Neurospora crassa. Mol Cell Biol. 2004;24(6):2536-45. https://doi.org/10.1128/mcb.24.6.25362545.2004

27. Fagard M, Boutet S, Morel JB, Bellini C, Vaucheret H. AGO1, QDE-2, and RDE-1 are related proteins required for posttranscriptional gene silencing in plants, quelling in fungi, and RNA interference in animals. Proc Natl Acad Sci U S A. 2000;97(21):11650-4. https://doi.org/10.1073/pnas.200217597.

28. Maiti M, Lee HC, Liu Y. QIP, a putative exonuclease, interacts with the Neurospora argonaute protein and facilitates conversion of duplex siRNA into single strands. Genes Dev. 2007;21(5):590-600. https://doi.org/10.1101/gad.1497607.

29. Drinnenberg IA, Weinberg DE, Xie KT, Mower JP, Wolfe KH, Fink GR, et al. RNAi in budding yeast. Science. 2009;326(5952): 544-50. https://doi.org/10.1126/science.1176945.

30. Yadav V, Sun S, Billmyre RB, Thimmappa BC, Shea T, Lintner R, et al. RNAi is a critical determinant of centromere evolution in closely related fungi. Proc Natl Acad Sci U S A. 2018;115(12): 3108-13. https://doi.org/10.1073/pnas.1713725115.

31. Feretzaki M, Billmyre RB, Clancey SA, Wang X, Heitman J. Gene Network Polymorphism Illuminates Loss and Retention of Novel RNAi Silencing Components in the Cryptococcus Pathogenic Species Complex. PLoS Genet. 2016;12(3):e1005868. https://doi. org/10.1371/journal.pgen.1005868.
32. Moazed D. Rejoice-RNAi for yeast. Science. 2009;326(5952):5334. https://doi.org/10.1126/science.1182102.

33. Weick EM, Miska EA. piRNAs: from biogenesis to function. Development. 2014;141(18):3458-71. https://doi.org/10.1242/ dev.094037.

34. Lekka E, Hall J. Noncoding RNAs in disease. FEBS Lett. 2018;592(17):2884-900. https://doi.org/10.1002/1873-3468. 13182.

35. Schmitt AM, Chang HY. Long noncoding RNAs in cancer pathways. Cancer Cell. 2016;29(4):452-63. https://doi.org/10.1016/j. ccell.2016.03.010

36. Chacko N, Lin X. Non-coding RNAs in the development and pathogenesis of eukaryotic microbes. Appl Microbiol Biotechnol. 2013;97(18):7989-97. https://doi.org/10.1007/s00253-013-5160y.

37. Johnsson P, Lipovich L, Grander D, Morris KV. Evolutionary conservation of long non-coding RNAs; sequence, structure, function. Biochimica Et Biophysica Acta-General Subjects. 2014;1840(3): 1063-71. https://doi.org/10.1016/j.bbagen.2013.10.035.

38. Noviello TMR, Di Liddo A, Ventola GM, Spagnuolo A, D'Aniello $\mathrm{S}$, Ceccarelli M, et al. Detection of long non-coding RNA homology, a comparative study on alignment and alignment-free metrics. Bmc Bioinformatics. 2018;19:407. https://doi.org/10.1186/s12859018-2441-6.

39. Yin QF, Yang L, Zhang Y, Xiang JF, Wu YW, Carmichael GG, et al. Long noncoding RNAs with snoRNA ends. Mol Cell. 2012;48(2):219-30. https://doi.org/10.1016/j.molcel.2012.07.033.

40. Zhang Y, Yang L, Chen LL. Life without a tail: new formats of long noncoding RNAs. Int J Biochem Cell Biol. 2014;54:338-49. https://doi.org/10.1016/j.biocel.2013.10.009.

41. Chen LL. Linking Long noncoding RNA localization and function. Trends Biochem Sci. 2016;41(9):761-72. https://doi.org/10.1016/j. tibs.2016.07.003.

42. Han P, Chang CP. Long non-coding RNA and chromatin remodeling. RNA Biol. 2015;12(10):1094-8. https://doi.org/10.1080/ 15476286.2015.1063770.

43. Lapidot M, Pilpel Y. Genome-wide natural antisense transcription: coupling its regulation to its different regulatory mechanisms. EMBO Rep. 2006;7(12):1216-22. https://doi.org/10.1038/sj. embor.7400857.

44. Donaldson ME, Saville BJ. Natural antisense transcripts in fungi. Mol Microbiol. 2012;85(3):405-17. https://doi.org/10.1111/j. 1365-2958.2012.08125.x.

45. Wight $\mathrm{M}$, Werner $\mathrm{A}$. The functions of natural antisense transcripts. Role of Non-Cod RNAsBiol. 2013;54:91-101. https://doi.org/10. 1042/bse0540091.

46. Jesenicnik T, Stajner N, Radisek S, Jakse J. RNA interference core components identified and characterised in Verticillium nonalfalfae, a vascular wilt pathogenic plant fungi of hops. Sci Rep. 2019;9:8651. https://doi.org/10.1038/s41598-019-44494-8.

47. Jin Y, Zhao JH, Zhao P, Zhang T, Wang S, Guo HS. A fungal milRNA mediates epigenetic repression of a virulence gene in Verticillium dahliae. Philosophical Transactions of the Royal Society B-Biological Sciences. 2019;374:1767. https://doi.org/10. 1098/rstb.2018.0309. This paper provided a functional characterization on role of miRNA in chromatin remodelling and its role in fungal virulence.

48. Weinberg DE, Nakanishi K, Patel DJ, Bartel DP. The inside-out mechanism of dicers from budding yeasts. Cell. 2011;146(2):26276. https://doi.org/10.1016/j.cell.2011.06.021.

49. Lawrence M, Daujat S, Schneider R. Lateral thinking: how histone modifications regulate gene expression. Trends Genet. 2016;32(1): 42-56. https://doi.org/10.1016/j.tig.2015.10.007.

50. Karlic R, Chung HR, Lasserre J, Vlahovicek K, Vingron M. Histone modification levels are predictive for gene expression. 
Proc Natl Acad Sci U S A. 2010;107(7):2926-31. https://doi.org/ 10.1073/pnas.0909344107.

51. Richards EJ, Elgin SCR. Epigenetic codes for heterochromatin formation and silencing: rounding up the usual suspects. Cell. 2002;108(4):489-500. https://doi.org/10.1016/s0092-8674(02) 00644-x.

52. Allshire RC, Madhani HD. Ten principles of heterochromatin formation and function. Nat Rev Mol Cell Biol. 2018;19(4):229-44. https://doi.org/10.1038/nrm.2017.119.

53.• Zhang T, Zhao YL, Zhao JH, Wang S, Jin Y, Chen ZQ, et al. Cotton plants export microRNAs to inhibit virulence gene expression in a fungal pathogen. Nat Plants. 2016;2(10):16153. https://doi.org/10. 1038/nplants.2016.153. This paper showed that host can transfer sRNA cross kingdom to downregulate virulence.

54.• Cai Q, Qiao LL, Wang M, He BY, Lin FM, Palmquist J, et al. Plants send small RNAs in extracellular vesicles to fungal pathogen to silence virulence genes. Science. 2018;360(6393):1126-9. https:// doi.org/10.1126/science.aar4142. This paper showed that extracellular vesicles play an important role in cross-kingdom transfer of sRNAs, thus providing mechanism for this phenomenon.

55. Mathivanan S, Ji H, Simpson RJ. Exosomes: Extracellular organelles important in intercellular communication. J Proteome. 2010;73(10):1907-20. https://doi.org/10.1016/j.jprot.2010.06.006.

56. Cui C, Wang Y, Liu J, Zhao J, Sun P. Wang S. A fungal pathogen deploys a small silencing RNA that attenuates mosquito immunity and facilitates infection. Nat Commun. 2019;10(1):4298. https:// doi.org/10.1038/s41467-019-12323-1. This paper provided additional evidence on role of extracellular vesicles in cross kingdom sRNA transfer. This paper also highighted developmentally regulated dual role of fungal milRNA in disease initiation and fungal proliferation.

57. Hillyer JF. Insect immunology and hematopoiesis. Dev Comp Immunol. 2016;58:102-18. https://doi.org/10.1016/j.dci.2015.12. 006.

58. An CJ, Budd A, Kanost MR, Michel K. Characterization of a regulatory unit that controls melanization and affects longevity of mosquitoes. Cell Mol Life Sci. 2011;68(11):1929-39. https://doi.org/ 10.1007/s00018-010-0543-z.

59.• Weiberg A, Wang M, Lin FM, Zhao HW, Zhang ZH, Kaloshian I, et al. Fungal small rnas suppress plant immunity by hijacking host RNA interference pathways. Science. 2013;342(6154):118-23. https://doi.org/10.1126/science.1239705. This paper highlighted the role of cross kingdom sRNA transfer from pathogen to host to regulate virulence.

60. Croston TL, Lemons AR, Beezhold DH, Green BJ. MicroRNA Regulation of Host Immune Responses following Fungal Exposure. Front Immunol. 2018;9:170. https://doi.org/10.3389/ fimmu.2018.00170.

61. Bernstein DA, Vyas VK, Weinberg DE, Drinnenberg IA, Bartel DP, Fink GR. Candida albicans dicer (CaDcr1) is required for efficient ribosomal and spliceosomal RNA maturation. Proc Natl Acad Sci U S A. 2012;109(2):523-8. https://doi.org/10.1073/pnas. 1118859109

62. Liu H, Cottrell TR, Pierini LM, Goldman WE, Doering TL. RNA interference in the pathogenic fungus Cryptococcus neoformans. Genetics. 2002;160(2):463-70.

63. Mouyna I, Henry C, Doering TL, Latge JP. Gene silencing with RNA interference in the human pathogenic fungus Aspergillus fumigatus. FEMS Microbiol Lett. 2004;237(2):317-24. https:// doi.org/10.1016/j.femsle.2004.06.048.

64. Kadotani N, Nakayashiki H, Tosa Y, Mayama S. RNA silencing in the phytopathogenic fungus Magnaporthe oryzae. Mol PlantMicrobe Interact. 2003;16(9):769-76. https://doi.org/10.1094/ mpmi.2003.16.9.769.
65. Chen Y, Gao Q, Huang M, Liu Y, Liu Z, Liu X, et al. Characterization of RNA silencing components in the plant pathogenic fungus Fusarium graminearum. Sci Rep. 2015;5:12500. https://doi.org/10.1038/srep12500.

66. Chacko N, Zhao Y, Yang E, Wang L, Cai JJ, Lin X. The lncRNA RZE1 Controls Cryptococcal Morphological Transition. PLoS Genet. 2015;11(11):e1005692. https://doi.org/10.1371/journal. pgen.1005692.

67. Lin X, Jackson JC, Feretzaki M, Xue C, Heitman J. Transcription factors Mat2 and Znf2 operate cellular circuits orchestrating opposite- and samesex mating in Cryptococcus neoformans. PLoS Genet. 2010;6(5):e1000953. https://doi.org/10.1371/journal.pgen. 1000953.

68. Goulet KM, Storfie ERM, Saville BJ. Exploring links between antisense RNAs and pathogenesis in Ustilago maydis through transcript and gene characterization. Fungal Genet Biol. 2020;134: 103283. https://doi.org/10.1016/j.fgb.2019.103283. This paper characterized the role of natural antisense transcripts and fungal pathogenesis.

69. Donaldson ME, Saville BJ. Ustilago maydis natural antisense transcript expression alters mRNA stability and pathogenesis. Mol Microbiol. 2013;89(1):29-51. https://doi.org/10.1111/mmi.12254.

70. Morrison EN, Donaldson ME, Saville BJ. Identification and analysis of genes expressed in the Ustilago maydis dikaryon: uncovering a novel class of pathogenesis genes. Can J Plant Pathol-Revue Canadienne De Phytopathologie. 2012;34(3):41735. https://doi.org/10.1080/07060661.2012.697077.

71. Smith JE, Baker KE. Nonsense-mediated RNA decay - a switch and dial for regulating gene expression. Bioessays. 2015;37(6):612-23. https://doi.org/10.1002/bies.201500007.

72. Atkinson SR, Marguerat S, Bitton DA, Rodriguez-Lopez M, Rallis $\mathrm{C}$, Lemay JF, et al. Long noncoding RNA repertoire and targeting by nuclear exosome, cytoplasmic exonuclease, and RNAi in fission yeast. Rna. 2018;24(9):1195-213. https://doi.org/10.1261/rna. 065524.118

73. Wery M, Descrimes M, Vogt N, Dallongeville AS, Gautheret D, Morillon A. Nonsense-mediated decay restricts LncRNA levels in yeast unless blocked by double-stranded RNA structure. Mol Cell. 2016;61(3):379-92. https://doi.org/10.1016/j.molcel.2015.12.020.

74. Wang YL, Shao Y, Zhu YL, Wang K, Ma B, Zhou QM, et al. XRN1-associated long non-coding RNAs may contribute to fungal virulence and sexual development in entomopathogenic fungus Cordyceps militaris. Pest Manag Sci. 2019;75(12):3302-11. https://doi.org/10.1002/ps.5453.

75. Donaldson ME, Ostrowski LA, Goulet KM, Saville BJ. Transcriptome analysis of smut fungi reveals widespread intergenic transcription and conserved antisense transcript expression. BMC Genomics. 2017;18:340. https://doi.org/10.1186/ s12864-017-3720-8.

76. Laurie JD, Linning R, Bakkeren G. Hallmarks of RNA silencing are found in the smut fungus Ustilago hordei but not in its close relative Ustilago maydis. Curr Genet. 2008;53(1):49-58. https://doi.org/10. 1007/s00294-007-0165-7.

77. Smith CA, Robertson D, Yates B, Nielsen DM, Brown D, Dean RA, et al. The effect of temperature on natural antisense transcript (NAT) expression in Aspergillus flavus. Curr Genet. 2008;54(5): 241-69. https://doi.org/10.1007/s00294-008-0215-9.

78. Abdel-Hadi A, Carter D, Magan N. Temporal monitoring of the nor-1 (aflD) gene of Aspergillus flavus in relation to aflatoxin B-1 production during storage of peanuts under different water activity levels. J Appl Microbiol. 2010;109(6):1914-22. https://doi.org/10. 1111/j.1365-2672.2010.04820.x.

79. Schmidt-Heydt M, Abdel-Hadi A, Magan N, Geisen R. Complex regulation of the aflatoxin biosynthesis gene cluster of Aspergillus flavus in relation to various combinations of water activity and 
temperature. Int J Food Microbiol. 2009;135(3):231-7. https://doi. org/10.1016/j.ijfoodmicro.2009.07.026.

80. Liu K, Gao L, Ma X, Huang JJ, Chen J, Zeng L, et al. Long noncoding RNAs regulate drug resistance in cancer. Mol Cancer. 2020;19(1):54. https://doi.org/10.1186/s12943-020-01162-0.

81.• Calo S, Shertz-Wall C, Lee SC, Bastidas RJ, Nicolas FE, Granek JA, et al. Antifungal drug resistance evoked via RNAi-dependent epimutations. Nature. 2014;513(7519):555. https://doi.org/10. 1038 /nature 13575. This paper was the first to show epimutations based on endogenous gene that regulate drug response by an RNAi mechanism.

82. Chang Z, Billmyre RB, Lee SC, Heitman J. Broad antifungal resistance mediated by RNAi-dependent epimutation in the basal human fungal pathogen Mucor circinelloides. PLoS Genet. 2019;15(2): e1007957. https://doi.org/10.1371/journal.pgen.1007957. This paper further characterized role of transient epimutations to adapt to stress against a different class showing broader roles of RNAi in stress response and better environmental adaptation.

83. Torres-Martinez S, Ruiz-Vazquez RM. RNAi pathways in Mucor: a tale of proteins, small RNAs and functional diversity. Fungal Genet Biol. 2016;90:44-52. https://doi.org/10.1016/j.fgb.2015.11.006.

84. Bastidas RJ, Shertz CA, Lee SC, Heitman J, Cardenas ME. Rapamycin exerts antifungal activity in vitro and in vivo against Mucor circinelloides via FKBP12-dependent inhibition of Tor. Eukaryot Cell. 2012;11(3):270-81. https://doi.org/10.1128/ec. 05284-11.

85. Chang Z, Yadav V, Lee SC, Heitman J. Epigenetic mechanisms of drug resistance in fungi. Fungal Genet Biol. 2019;132:103253. https://doi.org/10.1016/j.fgb.2019.103253.

86.• Ard R, Tong P, Allshire RC. Long non-coding RNA-mediated transcriptional interference of a permease gene confers drug tolerance in fission yeast. Nat Commun. 2014;5:5576. https://doi.org/ 10.1038 /ncomms6576. This paper showed the role of IncRNA in drug reponse by chromatin remodelling.
87. Rhind N, Chen ZH, Yassour M, Thompson DA, Haas BJ, Habib N, et al. Comparative functional genomics of the fission yeasts. Science. 2011;332(6032):930-6. https://doi.org/10.1126/science. 1203357.

88. Ning BT, Yu DK, Yu AM. Advances and challenges in studying noncoding RNA regulation of drug metabolism and development of RNA therapeutics. Biochem Pharmacol. 2019;169:113638. https://doi.org/10.1016/j.bcp.2019.113638.

89. Hyland R, Jones BC, Smith DA. Identification of the cytochrome P450 enzymes involved in the N-oxidation of voriconazole. Drug Metab Dispos. 2003;31(5):540-7. https://doi.org/10.1124/dmd.31. 5.540 .

90. Long Y, Wang X, Youmans DT, Cech TR. How do lncRNAs regulate transcription? Sci Adv. 2017;3(9):eaao2110. https://doi. org/10.1126/sciadv.aao2110.

91. Joh RI, Palmieri CM, Hill IT, Motamedi M. Regulation of histone methylation by noncoding RNAs. Biochimica Et Biophysica ActaGene Regulatory Mechanisms. 2014;1839(12):1385-94. https:// doi.org/10.1016/j.bbagrm.2014.06.006.

92. Jochl C, Rederstorff M, Hertel J, Stadler PF, Hofacker IL, Schrettl $\mathrm{M}$, et al. Small ncRNA transcriptome analysis from Aspergillus fumigatus suggests a novel mechanism for regulation of protein synthesis. Nucleic Acids Res. 2008;36(8):2677-89. https://doi. org/10.1093/nar/gkn123.

93. Rosenberg A, Ene IV, Bibi M, Zakin S, Segal ES, Ziv N, et al. Antifungal tolerance is a subpopulation effect distinct from resistance and is associated with persistent candidemia. Nat Commun. 2018;9(1):2470. https://doi.org/10.1038/s41467-018-04926-x.

94. Windels EM, Michiels JE, Van den Bergh B, Fauvart M, Michiels J. Antibiotics: Combatting Tolerance To Stop Resistance. Mbio. 2019;10(5):e02095-19. https://doi.org/10.1128/mBio.02095-19.

Publisher's Note Springer Nature remains neutral with regard to jurisdictional claims in published maps and institutional affiliations. 